\title{
THE MATHEMATICAL MODELING AND INVESTIGATION OF THE STRESS-STRAIN STATE OF THE THREE-LAYER THERMOSENSITIVE HOLLOW CYLINDER
}

\author{
Iryna RAKOCHA*, Vasyl POPOVYCH* \\ *Lviv Polytechnic National University, 12 Bandera street, Lviv, Ukraine \\ "Pidstryhach Institute for Applied Problems of Mechanics and Mathematics of NASU, 3-b Naukova street, Lviv, Ukraine \\ irinka.rakocha@gmail.com, dept19@iapmm.lviv.ua
}

received 9 July 2015, revised 14 July 2016, accepted 18 July 2016

\begin{abstract}
Stationary temperature distribution in a three-layer infinite hollow cylinder based on the thermosensitive body model was determined. The cylinder is subjected to the steady temperature on the inner surface and on the outer one is present the convective heat exchange. In the second layer exist heat sources with parabolic dependence on radial coordinate. The components of the thermostressed state have been found. The influence of the temperature dependence of the thermal and mechanical components characteristics of materials on the temperature distribution has been investigated.
\end{abstract}

Key words: Three-Layered Cylinder, Thermosensitive Material, Temperature, Thermostressed State

\section{INTRODUCTION}

Knowledge of the components of the thermostressed state of structural elements, including hollow cylinders (Aziz, 2013; Argeso and Eraslan, 2008, Bazarenko, 2008; Fazelli et al., 2013; Shi et al., 2007; Zhu, 2002), subjected to given power and thermal loads underlie calculation on durability and reliability. The appropriate definition of temperature field and caused by it stress-strain state is based on the model of the thermosensitive body (Kushnir and Popovych, 2009; 2011), which takes into account the dependence of thermal and mechanical characteristics and parameters of heat exchange (heat transfer coefficients, degrees of surface blackness) on temperature are performed according to their application under condition of high-temperature heating.

The detailed review of studies relating to the definition of thermostressed state of thermosensitive bodies from the beginning of the investigation of the problem to its current state was provided in articles (Kushnir and Popovych, 2009; Noda, 1991). Papers (Carslaw and Jaeger, 1996; Goto and Suzuki, 1996; Grzes, 2010; Kushnir and Popovych, 2009; 2011; Kushnir and Protsiuk, 2010; Lee, 1998; Noda, 1991; Och, 2014; 2015; Popovych and Kalynyak, 2014; Shariyat, 2007, Shen, 2001; Tanigava and Akai, 1996; Yevtushenko and Kuciej, 2014; Zhu and Chao, 2002) represent mathematical models of thermostressed multilayer structures, which take into account the dependence of thermomechanical characteristics of the layer materials on the temperature as well as the new methods of their solution. In papers (Carslaw and Jaeger, 1996; Kushnir and Popovych, 2009; 2011; Kushnir and Protsiuk, 2010; Noda, 1991; Och, 2014; 2015; Popovych and Kalynyak, 2014; Zhu and Chao, 2002) the importance to take into account the thermosensitivity of structural components at investigation the temperature and stress distribution is indicated.

In the article the stationary temperature distribution in ele- ments of microelectronics, which are modeled by three-layer hollow thermosensitive cylinder with heat sources in second layer, constant temperature on the inner surface of the cylinder and convective heat exchange with the environment on the outer one, is determined. The components of the stress-stain state have been defined. The influence of the thermosensitivity of materials on the temperature distribution and the distribution of the components of stress-strain state has been investigated.

\section{THE FORMULATION OF THE PROBLEM}

The three-layer infinite thermosensitive hollow cylinder is considered. Each layer was made of different material. The cylinder is heated by heat sources which are located in the middle layer and are distributed by parabolic law

$W^{(2)}(r)=-\frac{4 W_{0}^{(2)}}{\left(r_{3}-r_{2}\right)^{2}}\left(r-r_{2}\right)\left(r-r_{3}\right)$.

The temperature is constant $t=t_{\text {in }}$ on inner surface $r=r_{1}$ and the convective heat exchange with the environment on outer one $r=r_{4}$ is present. The coefficient of heat transfer through this surface is constant and equal to $\alpha$. The conditions of ideal thermal contact between neighbour layers are satisfied. Let us determine the temperature distribution and the distribution of components of the cylinder stress-strain state at mentioned conditions.

\section{THE MATHEMATICAL MODEL FOR THE DETERMINATION OF TEMPERATURE DISTRIBUTION}

It includes:

- the heat conductivity equations for each layer 
$\frac{1}{r} \frac{d}{d r}\left(r \lambda_{t}^{(i)}\left(t_{i}\right) \frac{d t_{i}}{d r}\right)=W^{(i)}(r), r_{i}<r<r_{i+1}, i=\overline{1,3}$

- boundary conditions on the boundary surfaces

$\left.t_{1}\right|_{r=r_{1}}=t_{\text {in }}, \quad\left[\lambda_{t}^{(3)}\left(t_{3}\right) \frac{d t_{3}}{d r}+\alpha\left(t_{3}-t_{\text {out }}\right)\right]_{r=r_{4}}=0$,

- contact conditions on the neighbouring surfaces of the cylinder

$\left.t_{1}\right|_{r=r_{2}}=\left.t_{2}\right|_{r=r_{2}},\left.\quad t_{2}\right|_{r=r_{3}}=\left.t_{3}\right|_{r=r_{3}}$,

$\left.\lambda_{t}^{(1)}\left(t_{1}\right) \frac{d t_{1}}{d r}\right|_{r=r_{2}}=\left.\lambda_{t}^{(2)}\left(t_{2}\right) \frac{d t_{2}}{d r}\right|_{r=r_{2}}$,

$\left.\lambda_{t}^{(2)}\left(t_{2}\right) \frac{d t_{2}}{d r}\right|_{r=r_{3}}=\left.\lambda_{t}^{(3)}\left(t_{3}\right) \frac{d t_{3}}{d r}\right|_{r=r_{3}}$.

Model (1)-(4) is nonlinear due to the dependence of thermal conductivity coefficients $\lambda_{t}^{(i)}\left(t_{i}\right)(i=\overline{1,3})$ on temperature. Those dependencies are usually given in reference books and are represented in the form of tables.

To conduct theoretic reserch work it is convenient to present the thermal conductivity coefficients as analytical expression $\lambda_{t}^{(i)}=\lambda_{t}^{(i)}\left(t_{i}\right)$ with the purpose to conduct theoretic research work. To achieve such dependencies the tabular function is approximated with some functional dependence. The least squares method is an example of possible technique to be used with such approximation in order to determine the unknown parameters.

Let the thermal conductivity coefficients for each cylinder component to be in the temperature range $\left[t_{b} ; t_{e}\right]$. We are going to describe them with quite common linear dependencies $\lambda_{t}^{(i)}=a_{i} t_{i}+b_{i}$. Parameters $a_{i}$ and $b_{i}$ are unknown. According to the method of least squares they are selected so that the sum of the differences' squares between experimental and theoretical values will bw minimal.

Let us represent the thermal conductivity coefficients of cylinder layers in the form $\lambda_{t}^{(i)}\left(t_{i}\right)=\lambda_{t 0}^{(i)} \lambda_{t}^{(i) *}\left(T_{i}\right)$, where the values $\lambda_{\text {to }}^{(i)}$ have the dimensionality of thermal conductivity coefficient and $\lambda_{t}^{(i) *}\left(T_{i}\right)$ is dimensionless function of dimensionless temperature $\mathrm{T}_{\mathrm{i}}=\mathrm{t}_{\mathrm{i}} / \mathrm{t}_{\mathrm{e}}$, (where the temperature $\mathrm{t}_{\mathrm{e}}$ is chosen as a measuring indicator). Due to such linear dependence of the thermal conductivity coefficient on the temperature such representations will have the following form

$\lambda_{\mathrm{t}}^{(\mathrm{i})}\left(\mathrm{t}_{\mathrm{i}}\right)=\lambda_{\mathrm{t} 0}^{(\mathrm{i})}\left(1+\mathrm{k}_{\mathrm{i}}\left(\mathrm{T}_{\mathrm{i}}-\mathrm{T}_{\mathrm{b}}\right)\right)$,

where $\mathrm{T}_{\mathrm{b}}=\mathrm{t}_{\mathrm{b}} / \mathrm{t}_{\mathrm{e}}, \mathrm{k}_{\mathrm{i}}=\mathrm{a}_{\mathrm{i}} \mathrm{t}_{\mathrm{e}} /\left(\mathrm{a}_{\mathrm{i}} \mathrm{t}_{\mathrm{b}}+\mathrm{b}_{\mathrm{i}}\right), \mathrm{a} \lambda_{\mathrm{t} 0}^{(\mathrm{i})}=\mathrm{a}_{\mathrm{i}} \mathrm{t}_{\mathrm{b}}+\mathrm{b}_{\mathrm{i}}$.

Let us introduce the characteristic length value $l_{0}$ and dimensionless coordinate $\rho=\mathrm{r} / \mathrm{l}_{0}$ to present the mathematical model (1)-(4) in dimensionless form:

$\frac{d}{d \rho}\left(\rho \lambda_{t}^{(i) *}\left(T_{i}\right) \frac{d T_{i}}{d \rho}\right)=W^{(i)}(\rho), \rho_{i}<\rho<\rho_{i+1}, i=\overline{1,3}$,

$\left.T_{1}\right|_{\rho=\rho_{1}}=T_{\text {in }},\left[\lambda_{t}^{(3) *}\left(T_{3}\right) \frac{d T_{3}}{d \rho}+B i\left(T_{3}-T_{\text {out }}\right)\right]_{\rho=\rho_{4}}=0$,

$\left.T_{1}\right|_{\rho=\rho_{2}}=\left.T_{2}\right|_{\rho=\rho_{2}},\left.\quad T_{2}\right|_{\rho=\rho_{3}}=\left.T_{3}\right|_{\rho=\rho_{3}}$,

$$
\begin{aligned}
& \left.\lambda_{t}^{(1) *}\left(T_{1}\right) \frac{d T_{1}}{d \rho}\right|_{\rho=\rho_{2}}=\left.K_{\lambda}^{(2)} \lambda_{t}^{(2) *}\left(T_{2}\right) \frac{d T_{2}}{d \rho}\right|_{\rho=\rho_{2}}, \\
& \left.\lambda_{t}^{(2) *}\left(T_{2}\right) \frac{d T_{2}}{d \rho}\right|_{\rho=\rho_{3}}=\left.K_{\lambda}^{(3)} \lambda_{t}^{(3)}\left(T_{3}\right) \frac{d T_{3}}{d \rho}\right|_{\rho=\rho_{3}} .
\end{aligned}
$$

where: $\mathrm{T}_{\text {in }}=\mathrm{t}_{\text {in }} / \mathrm{t}_{\mathrm{e}}, \mathrm{T}_{\text {out }}=\mathrm{t}_{\text {out }} / \mathrm{t}_{\mathrm{e}}, \mathrm{K}_{\lambda}^{(\mathrm{j})}=\lambda_{\mathrm{t} 0}^{(\mathrm{j})} / \lambda_{\mathrm{t} 0}^{(\mathrm{j}-1)}(\mathrm{j}=$ $2,3), W^{(1)}(\rho)=W^{(3)}(\rho)=0, W^{(2)}(\rho)=-\frac{4 \cdot P o}{\left(\rho_{3}-\rho_{2}\right)^{2}} \rho(\rho-$ $\left.\rho_{2}\right)\left(\rho-\rho_{3}\right)$, Po $=W_{0}^{(2)} l_{0}^{2} /\left(\lambda_{t 0}^{(2)} t_{e}\right)$ is the Pomerantsev number, $\mathrm{Bi}=\alpha \mathrm{l}_{0} / \lambda_{\mathrm{t} 0}^{(3)}$ is the Biot number.

\section{THE CONSTRUCTION OF THE SOLUTION OF THE MATHEMATICAL MODEL}

To construct the solution of the problem (6)-(9) the Kirchhoff variables are introduced:

$\theta_{i}=\int_{T_{b}}^{T_{i}} \lambda_{t}^{(i) *}\left(T_{i}\right) d T_{i}, \quad i=\overline{1,3}$.

The following problem relative to mentioned above variables was received

$\frac{d}{d \rho}\left(\rho \frac{d \theta_{i}}{d \rho}\right)=W^{(i)}(\rho), \rho_{i}<\rho<\rho_{i+1}, i=\overline{1,3}$,

$\left.\theta_{1}\right|_{\rho=\rho_{1}}=\theta_{\text {in }},\left[\frac{d \theta_{3}}{d r}+B i\left(T_{3}\left(\theta_{3}\right)-T_{\text {out }}\right)\right]_{\rho=\rho_{4}}=0$,

$\left.T_{1}\left(\theta_{1}\right)\right|_{\rho=\rho_{2}}=\left.T_{2}\left(\theta_{2}\right)\right|_{\rho=\rho_{2}},\left.T_{2}\left(\theta_{2}\right)\right|_{\rho=\rho_{3}}=\left.T_{3}\left(\theta_{3}\right)\right|_{\rho=\rho_{3}}$,

$\left.\frac{d \theta_{1}}{d \rho}\right|_{\rho=\rho_{2}}=\left.K_{\lambda}^{(2)} \frac{d \theta_{2}}{d \rho}\right|_{\rho=\rho_{2}},\left.\frac{d \theta_{2}}{d \rho}\right|_{\rho=\rho_{3}}=\left.K_{\lambda}^{(3)} \frac{d \theta_{3}}{d \rho}\right|_{\rho=\rho_{3}}$,

where:

$\theta_{\text {in }}=\int_{T_{b}}^{T_{\text {in }}} \lambda_{t}^{(1) *}\left(T_{1}\right) d T_{1}=\left(T_{\text {in }}-T_{b}\right)+\frac{k_{1}}{2}\left(T_{\text {in }}-T_{b}\right)^{2}$.

As the result of the Kirchhoff transformation we have got a simpler problem in which the nonlinearity is present in 2nd condition (12) and (13). According to the linear dependence of the coefficients of thermal conductivity on temperature (5) as the consequence of formulas (10):

$\theta_{i}=\left(T_{i}-T_{b}\right)+\frac{k_{i}}{2}\left(T_{i}-T_{b}\right)^{2}$

we have

$T_{i}\left(\theta_{i}\right)=\frac{\sqrt{1+2 k_{i} \theta_{i}}-1}{k_{i}}+T_{b}$.

Let us note that the selected sign before the square root in temperature expressions provides the physical sense.

After integrating the equations (11) we find that:

$\frac{d \theta_{1}}{d \rho}=\frac{C_{11}}{\rho}, \quad \frac{d \theta_{2}}{d \rho}=\frac{C_{21}}{\rho}+\widetilde{W}^{(2)}(\rho), \quad \frac{d \theta_{3}}{d \rho}=\frac{C_{31}}{\rho}$, 
$\theta_{1}=C_{11} \ln \frac{\rho}{\rho_{1}}+C_{12}$

$\theta_{2}=C_{21} \ln \frac{\rho}{\rho_{2}}+C_{22}+\bar{W}^{(2)}(\rho)$,

$\theta_{3}=C_{31} \ln \frac{\rho}{\rho_{3}}+C_{32}$,

where:

$$
\begin{aligned}
\widetilde{W}^{(2)}(\rho)= & -\frac{4 \cdot P o}{\left(\rho_{3}-\rho_{2}\right)^{2}}\left(\frac{\rho^{3}}{4}-\frac{\rho^{2}}{3}\left(\rho_{3}+\rho_{2}\right)+\frac{\rho}{2} \rho_{2} \rho_{3}+\frac{\rho_{2}^{3}}{6 \rho}\right. \\
& \left.\cdot\left(\rho_{3}-\frac{\rho_{2}}{2}\right)\right) \\
\bar{W}^{(2)}(\rho)= & -\frac{4 \cdot P o}{\left(\rho_{3}-\rho_{2}\right)^{2}}\left(\rho^{2}\left(\frac{\rho^{2}}{16}-\rho\left(\rho_{3}+\rho_{2}\right)+\frac{\rho_{2} \rho_{3}}{4}\right)-\rho_{2}^{3}\right. \\
& \left.\cdot\left(\frac{\rho_{2}}{16}-\frac{\rho_{3}+\rho_{2}}{9}+\frac{\rho_{3}}{4}\right)-\frac{\rho_{2}^{3}}{6} \cdot \ln \frac{\rho}{\rho_{2}} \cdot\left(\rho_{3}-\frac{\rho_{2}}{2}\right)\right) .
\end{aligned}
$$

The solutions (17) contain 6 unknown constants of integration. To find them let us suppose the constants in the enpression for Kirchhoff variables to be known (the basic ones) in the first layer $\mathrm{C}_{11}$ and $\mathrm{C}_{12}$, for example. The remaining constants are expressed through basic ones using the contact conditions on the neighbour surface in the cylinder. Herewith the equvivalent conditions (Popovych V.S. and Kalynyak B. M., 2014) instead of conditions (13) are used:

$\theta_{i+1}-\left.\theta_{i}\right|_{\rho=\rho_{i+1}}=\left.\frac{k_{i+1}-k_{i}}{2}\left(T_{i}\left(\theta_{i}\right)-T_{b}\right)^{2}\right|_{\rho=\rho_{i+1}}$,

$i=1,2$.

From the conditions (14) and (18) we obtain correspondingly: $C_{21}=C_{11} / K_{\lambda}^{(2)}, C_{31}=\left(C_{11} / K_{\lambda}^{(2)}+\rho_{3} \widetilde{W}^{(2)}\left(\rho_{3}\right)\right) / K_{\lambda}^{(3)}$, $C_{22}=C_{11} \ln \frac{\rho_{2}}{\rho_{1}}+C_{12}+\frac{k_{2}-k_{1}}{2}$

$$
\cdot\left(\left(\sqrt{1+2 k_{1}\left(C_{11} \ln \frac{\rho_{2}}{\rho_{1}}+C_{12}\right)}-1\right) / k_{1}\right)^{2},
$$

$C_{32}=\left.\theta_{2}\right|_{\rho=\rho_{3}}+\frac{k_{3}-k_{2}}{2}\left(\left(\sqrt{1+\left.2 k_{2} \theta_{2}\right|_{\rho=\rho_{3}}}-1\right) / k_{2}\right)^{2}$,

where:

$\left.\theta_{2}\right|_{\rho=\rho_{3}}$

$=C_{11}\left(\ln \frac{\rho_{2}}{\rho_{1}}+\ln \frac{\rho_{3}}{\rho_{2}} / K_{\lambda}^{(2)}\right)+C_{12}+\bar{W}^{(2)}\left(\rho_{3}\right)$

$+\frac{k_{2}-k_{1}}{2}\left(\left(\sqrt{1+2 k_{1}\left(C_{11} \ln \frac{\rho_{2}}{\rho_{1}}+C_{12}\right)}-1\right) / k_{1}\right)^{2}$.

The constant of integration $\mathrm{C}_{\mathrm{i} 1}$ and $\mathrm{C}_{\mathrm{i} 2}(\mathrm{i}=2,3)$ are expressed through the basic ones $C_{11}$ and $C_{12}$, which are found from boundary conditions (12). Using the first one we receive

$C_{12}=\left(T_{\text {in }}-T_{b}\right)+\frac{k_{1}}{2}\left(T_{\text {in }}-T_{b}\right)^{2}$.

From the second condition of (12) the algebraic equation was received

$\frac{C_{31}}{\rho_{4}}+B i\left(\frac{\sqrt{1+\left.2 k_{3} \theta_{3}\right|_{\rho=\rho_{4}}}-1}{k_{3}}+T_{b}-T_{\text {out }}\right)=0$

to determine the constant $\mathrm{C}_{11}$.
The solution of (19) was found by software for analytical transformations. Using the obtained analytical expression for $\mathrm{C}_{11}$, which is not presented because of its complexity, we find automatically expressions of others constants of integration. Knowing $\mathrm{C}_{\mathrm{ij}}(\mathrm{i}=\overline{1,3}, \mathrm{j}=1,2)$ the temperature distributions of considered cylinder is given by formulas (16), (17).

\section{THE DETERMINATION OF THE TEMPERATURE DISTRIBUTION IN THE HOLLOW NONTHERMOSENSITIVE CYLINDER}

The dimensionless mathematical model for determining the temperature of a similar nonthermosensitive cylinder has the form

$\frac{d}{d \rho}\left(\rho \frac{d T_{i N}}{d \rho}\right)=W_{N}^{(i)}(\rho), \quad \rho_{i}<\rho<\rho_{i+1}, \quad i=\overline{1,3}$,

$\left.T_{1 N}\right|_{\rho=\rho_{1}}=T_{\text {in }},\left[\frac{d T_{3 N}}{d \rho}+B i_{N}\left(T_{3 N}-T_{\text {out }}\right)\right]_{\rho=\rho_{4}}=0$,

$\left.T_{1 N}\right|_{\rho=\rho_{2}}=\left.T_{2 N}\right|_{\rho=\rho_{2}},\left.\quad T_{2 N}\right|_{\rho=\rho_{3}}=\left.T_{3 N}\right|_{\rho=\rho_{3}}$,

$\left.\frac{d T_{1 N}}{d \rho}\right|_{\rho=\rho_{2}}=\left.K_{\lambda N}^{(2)} \frac{d T_{2 N}}{d \rho}\right|_{\rho=\rho_{2}}$,

$\left.\frac{d T_{2 N}}{d \rho}\right|_{\rho=\rho_{3}}=\left.K_{\lambda N}^{(3)} \frac{d T_{3 N}}{d \rho}\right|_{\rho=\rho_{3}}$,

where: $T_{i N}=t_{i N} / t_{e}, K_{\lambda N}^{(j)}=\lambda_{t N}^{(j)} / \lambda_{t N}^{(j-1)}(j=2,3), W_{N}^{(1)}(\rho)=$ $W_{N}^{(3)}(\rho)=0, \quad W_{N}^{(2)}(\rho)=-\frac{4 \cdot P o_{N}}{\left(\rho_{3}-\rho_{2}\right)^{2}} \cdot \rho\left(\rho-\rho_{2}\right)\left(\rho-\rho_{3}\right)$, $P o_{N}=P o \cdot \lambda_{t N}^{(2)} / \lambda_{t N}^{(2)}$ is the Pomerantsev number, $B i_{N}=$ $B i \cdot \lambda_{t N}^{(3)} / \lambda_{t N}^{(3)}$ is the Biot number.

From the problem (20)-(23) we find the following

$\frac{d T_{1 N}}{d \rho}=\frac{\bar{C}_{11}}{\rho}, \quad \frac{d T_{2 N}}{d \rho}=\frac{\bar{C}_{21}}{\rho}+\widetilde{W}_{N}^{(2)}(\rho), \quad \frac{d T_{3 N}}{d \rho}=\frac{\bar{C}_{31}}{\rho}$,

$T_{1 N}=\bar{C}_{11} \ln \frac{\rho}{\rho_{1}}+\bar{C}_{12}$,

$T_{2 N}=\bar{C}_{21} \ln \frac{\rho}{\rho_{2}}+\bar{C}_{22}+\bar{W}_{N}^{(2)}(\rho)$

$T_{3 N}=\bar{C}_{31} \ln \frac{\rho}{\rho_{3}}+\bar{C}_{32}$,

where:

$$
\begin{aligned}
\widetilde{W}_{N}^{(2)}(\rho)= & -\frac{4 \cdot P o_{N}}{\left(\rho_{3}-\rho_{2}\right)^{2}}\left(\frac{\rho^{3}}{4}-\frac{\rho^{2}}{3}\left(\rho_{3}+\rho_{2}\right)+\frac{\rho}{2} \rho_{2} \rho_{3}+\frac{\rho_{2}^{3}}{6 \rho}\right. \\
& \left.\cdot\left(\rho_{3}-\frac{\rho_{2}}{2}\right)\right),
\end{aligned}
$$

$$
\begin{aligned}
\bar{W}_{N}^{(2)}(\rho)= & -\frac{4 \cdot P o_{N}}{\left(\rho_{3}-\rho_{2}\right)^{2}}\left(\rho^{2}\left(\frac{\rho^{2}}{16}-\rho\left(\rho_{3}+\rho_{2}\right)+\frac{\rho_{2} \rho_{3}}{4}\right)-\rho_{2}^{3}\right. \\
& \left.\cdot\left(\frac{\rho_{2}}{16}-\frac{\rho_{3}+\rho_{2}}{9}+\frac{\rho_{3}}{4}\right)-\frac{\rho_{2}^{3}}{6} \ln \frac{\rho}{\rho_{2}}\left(\rho_{3}-\frac{\rho_{2}}{2}\right)\right) .
\end{aligned}
$$

Then using the boundary conditions (21) and conditions of layer contact (22) we find constants of integration

$\bar{C}_{11}=\left(K_{\lambda N}^{(2)}\left(\widetilde{W}_{N}^{(2)}\left(\rho_{3}\right) \rho_{3}-K_{\lambda N}^{(3)}\left(\bar{W}_{N}^{(2)}\left(\rho_{3}\right)+T_{i n}\right)\right)\right) / \Delta$, 


$$
\begin{aligned}
& \bar{C}_{12}= T_{\text {in }} \\
& \bar{C}_{21}=\left(\widetilde{W}_{N}^{(2)}\left(\rho_{3}\right) \rho_{3}-K_{\lambda N}^{(3)}\left(\bar{W}_{N}^{(2)}\left(\rho_{3}\right)+T_{\text {in }}\right)\right) / \Delta, \\
& \bar{C}_{22}= \frac{1}{\Delta}\left(\rho_{3} \cdot \widetilde{W}_{N}^{(2)}\left(\rho_{3}\right)-K_{\lambda N}^{(3)} \cdot\left(\bar{W}_{N}^{(2)}\left(\rho_{3}\right)+T_{\text {in }}\right)\right) \\
& \quad \cdot\left(\frac{1}{K_{\lambda N}^{(3)}}-\ln \frac{\rho_{3}}{\rho_{2}}\right), \\
& \bar{C}_{31}= \frac{1}{\Delta}\left(\frac{\rho_{3} \widetilde{W}_{N}^{(2)}\left(\rho_{3}\right)}{K_{\lambda N}^{(3)}}-\bar{W}_{N}^{(2)}\left(\rho_{3}\right)-T_{\text {in }}\right)+\frac{\widetilde{W}_{N}^{(2)}\left(\rho_{3}\right)}{K_{\lambda N}^{(3)}}, \\
& \bar{C}_{32}=\left(\frac{1}{\Delta}\left(\bar{W}_{N}^{(2)}\left(\rho_{3}\right)+T_{\text {in }}-\frac{\rho_{3} \widetilde{W}_{N}^{(2)}\left(\rho_{3}\right)}{K_{\lambda N}^{(3)}}\right)-\frac{\widetilde{W}_{N}^{(2)}\left(\rho_{3}\right)}{K_{\lambda N}^{(3)}}\right)\left(\frac{1}{\rho_{4} B i_{N}}\right. \\
&\left.\quad-\ln \frac{\rho_{3}}{\rho_{2}}\right)-T_{\text {out }}, \\
& \Delta=\ln \frac{\rho_{2}}{\rho_{1}}\left(1-K_{\lambda N}^{(3)} \ln \frac{\rho_{3}}{\rho_{2}}\right) .
\end{aligned}
$$

\section{THE DETERMINATION OF STRESS-STRAIN STATE}

If the elastic modulus $E^{(i)}\left(t_{i}\right)$, the Poisson ratio $v^{(i)}\left(t_{i}\right)$ and the temperature coefficients of the linear thermal expansions $\alpha_{t}^{(i)}\left(t_{i}\right)$ of cylinder components are represented in the form $\chi^{(\mathrm{i})}\left(\mathrm{t}_{\mathrm{i}}\right)=\chi_{0}^{(\mathrm{i})} \chi_{\mathrm{i}}^{*}\left(\mathrm{~T}_{\mathrm{i}}\right)$, where $\chi_{0}^{(\mathrm{i})}$ are the dimensional quantities equal to the value of the characteristic with the temperature $t_{b}$, and values $\chi_{\mathrm{i}}^{*}\left(\mathrm{~T}_{\mathrm{i}}\right)$ are the dimensionless function of dimensionless temperature $\mathrm{T}_{\mathrm{i}}$, then $E^{(i)}\left(t_{i}\right)=E_{0}^{(i)} E_{i}^{*}\left(T_{i}\right), v^{(i)}\left(t_{i}\right)=$ $v_{0}^{(i)} v_{i}^{*}\left(T_{i}\right), \alpha_{t}^{(i)}\left(t_{i}\right)=\alpha_{t 0}^{(i)} \alpha_{t i}^{*}\left(T_{i}\right)$.

Radial $\sigma_{\mathrm{r}}^{(\mathrm{i})}$ circumferential $\sigma_{\varphi}^{(\mathrm{i})}$ and axial $\sigma_{\mathrm{z}}^{(\mathrm{i})}$ stresses, radial $\mathrm{e}_{\mathrm{r}}^{(\mathrm{i})}$ and circumferential $\mathrm{e}_{\varphi}^{(\mathrm{i})}$ deformations and also radial displacements $u_{r}^{(i)}$ are calculated using expressions (Popovych Kalynyak, 2014) without mass forces:

$\sigma_{r}^{(i)}(\rho)=\gamma_{1 r}^{(i)}(\rho) \sigma^{(1)}\left(\rho_{1}\right)+\gamma_{2 r}^{(i)}(\rho) e_{z}+\gamma_{0 r}^{(i)}(\rho)$,

$\sigma_{\varphi}^{(i)}(\rho)=\sigma^{(i)}(\rho)-\sigma_{r}^{(i)}(\rho)$,

$\sigma_{z}^{(i)}(\rho)=E^{(i)} e_{z}+v^{(i)} \sigma^{(i)}(\rho)-E^{(i)} \Phi^{(i)}\left(T_{i}\right)$,

$e_{r}^{(i)}(\rho)$

$=\left(\sigma_{r}^{(i)}(\rho)-v^{(i)}\left(\sigma_{\varphi}^{(i)}(\rho)+\sigma_{z}^{(i)}(\rho)\right)+E^{(i)} \Phi^{(i)}\left(T_{i}\right)\right) / E^{(i)}$,

$e_{\varphi}^{(i)}(\rho)$
$=\left(\sigma_{\varphi}^{(i)}(\rho)-v^{(i)}\left(\sigma_{r}^{(i)}(\rho)+\sigma_{z}^{(i)}(\rho)\right)+E^{(i)} \Phi^{(i)}\left(T_{i}\right)\right) / E^{(i)}$,

$u_{r}^{(i)}(\rho)=\rho \cdot e_{\varphi}^{(i)}(\rho)$,

where $\sigma^{(i)}=\sigma_{r}^{(i)}+\sigma_{\varphi}^{(i)}-$ are the total stresses calculated by the formula:

$\sigma^{(i)}(\rho)=\gamma_{10}^{(i)}(\rho) \sigma^{(1)}\left(\rho_{1}\right)+\gamma_{20}^{(i)}(\rho) e_{z}+\gamma_{00}^{(i)}(\rho)$,

$$
\begin{aligned}
& \gamma_{10}^{(i)}(\rho)=\frac{1}{\psi^{(i)}(\rho)} \\
& \cdot\left(\left(1-\delta_{1 i}\right) \cdot \gamma_{1 r}^{(i-1)}\left(\rho_{i}\right) \cdot \chi_{2}^{(i)}(\rho)\right. \\
& +\left(\left(1-\left(v^{(1)}\left(\rho_{1}\right)\right)^{2}\right) / E^{(1)}\left(\rho_{1}\right)+\left(1-\delta_{1 i}\right)\right. \\
& \cdot \sum_{k=1}^{i-1}\left(\int_{\rho_{k}}^{\rho_{k+1}} \gamma_{1 r}^{(k)}(\eta) \cdot\left(\varphi^{(k)}(\eta)\right)^{\prime} d \eta+\beta^{(k)}\right. \\
& \left.\left.\left.\cdot \gamma_{1 r}^{(k)}\left(\rho_{k+1}\right)\right)\right) \cdot \chi_{1}^{(i)}(\rho)\right) \\
& \gamma_{20}^{(i)}(\rho)=\frac{1}{\psi^{(i)}(\rho)} \\
& \cdot\left(v^{(i)}(\rho)-v^{(i)}\left(\rho_{i}\right)+\left(1-\delta_{1 i}\right) \gamma_{2 r}^{(i-1)}\left(\rho_{i}\right) \cdot \chi_{2}^{(i)}(\rho)\right. \\
& +\left(v^{(i)}\left(\rho_{i}\right)-v^{(1)}\left(\rho_{1}\right)+\left(1-\delta_{1 i}\right)\right. \\
& \cdot \sum_{k=1}^{i-1}\left(\int_{\rho_{k}}^{\rho_{k+1}} \gamma_{2 r}^{(k)}(\eta) \cdot\left(\varphi^{(k)}(\eta)\right)^{\prime} d \eta+\beta^{(k)}\right. \\
& \left.\left.\left.\cdot \gamma_{2 r}^{(k)}\left(\rho_{k+1}\right)\right)\right) \cdot \chi_{1}^{(i)}(\rho)\right) \\
& \gamma_{00}^{(i)}(\rho)=\frac{1}{\psi^{(i)}(\rho)}\left(\left(-\delta_{1 i} p_{1}+\left(1-\delta_{1 i}\right) \cdot \gamma_{0 r}^{(i-1)}\left(\rho_{i}\right)\right) \cdot \chi_{2}^{(i)}(\rho)\right. \\
& -F^{(i)}(\rho)+F^{(i)}\left(\rho_{i}\right) \\
& +\left(\left(1-\delta_{1 i}\right)\right. \\
& \cdot \sum_{k=1}^{i-1}\left(\int_{\rho_{k}}^{\rho_{k+1}} \gamma_{0 r}^{(k)}(\eta) \cdot\left(\varphi^{(k)}(\eta)\right)^{\prime} d \eta+\beta^{(k)}\right. \\
& \left.\left.\left.\cdot \gamma_{0 r}^{(k)}\left(\rho_{k+1}\right)\right)-F^{(i)}\left(\rho_{i}\right)\right) \chi_{1}^{(i)}(\rho)\right) \\
& \gamma_{1 r}^{(i)}(\rho)=\frac{1}{\rho^{2}}\left(\left(1-\delta_{1 i}\right) \cdot \rho_{i}^{2} \cdot \gamma_{1 r}^{(i-1)}\left(\rho_{i}\right)+\int_{\rho_{i}}^{\rho} \eta \gamma_{10}^{(i)}(\eta) d \eta\right), \\
& \gamma_{2 r}^{(i)}(\rho)=\frac{1}{\rho^{2}}\left(\left(1-\delta_{1 i}\right) \cdot \rho_{i}^{2} \cdot \gamma_{2 r}^{(i-1)}\left(\rho_{i}\right)+\int_{\rho_{i}}^{\rho} \eta \gamma_{20}^{(i)}(\eta) d \eta\right), \\
& \gamma_{0 r}^{(i)}(\rho)=\frac{1}{\rho^{2}}\left(-\rho_{1}^{2} \cdot p_{1} \cdot \delta_{1 i}+\left(1-\delta_{1 i}\right) \cdot \rho_{i}^{2} \cdot \gamma_{0 r}^{(i-1)}\left(\rho_{i}\right)\right. \\
& \left.+\int_{\rho_{i}}^{\rho} \eta \gamma_{00}^{(i)}(\eta) d \eta\right)
\end{aligned}
$$

$$
\chi_{1}^{(i)}(\rho)=1+\left(\frac{\rho-\rho_{i}}{2}\right)^{2} \cdot\left(\varphi^{(i)}(\rho)\right)^{\prime} \cdot \frac{\rho_{i}}{\rho^{2}} \cdot \frac{E^{(i)}\left(\rho_{i}\right)}{1-v^{(i)}\left(\rho_{i}\right)^{\prime}}
$$




$$
\begin{aligned}
& \chi_{2}^{(i)}(\rho)=\frac{\rho-\rho_{i}}{2}\left(\frac{\rho_{i}^{2}}{\rho^{2}} \cdot\left(\varphi^{(i)}(\rho)\right)^{\prime}+\left(\varphi^{(i)}\left(\rho_{i}\right)\right)^{\prime}\right), \\
& \psi^{(i)}(\rho)=\frac{1-\left(v^{(i)}\left(\rho_{i}\right)\right)^{2}}{E^{(i)}\left(\rho_{i}\right)}-\left(\frac{\rho-\rho_{i}}{2}\right)^{2} \frac{1}{\rho}\left(\varphi^{(i)}\left(\rho_{i}\right)\right)^{\prime}, \\
& \varphi^{(i)}(\rho)=\frac{1-v^{(i)}(\rho)}{E^{(i)}(\rho)}, \quad\left(\varphi^{(i)}(\rho)\right)^{\prime}=\frac{d}{d \rho}\left(\frac{1-v^{(i)}(\rho)}{E^{(i)}(\rho)}\right), \\
& \beta_{i}=\varphi^{(i+1)}\left(\rho_{i+1}\right)-\varphi^{(i)}\left(\rho_{i+1}\right), \\
& F^{i}(\rho)=\left(1-v^{(i)}(\rho)\right) \cdot \Phi^{(i)}\left(T_{i}(\rho)\right)-\left(1-v^{(1)}\left(\rho_{1}\right)\right) \\
& \text { - } \Phi^{(1)}\left(T_{1}\left(\rho_{1}\right)\right) \text {, } \\
& \Phi^{(i)}\left(T_{i}\right)=t_{0} \cdot \int_{T_{p}}^{T_{i}} \alpha_{t}^{(i)}\left(T_{i}\right) d T_{i}, \\
& \sigma^{(1)}\left(\rho_{1}\right)=\frac{c_{1} d_{22}-c_{2} d_{12}}{d_{11} d_{22}-d_{21} d_{12}}, \quad e_{z}=\frac{c_{2} d_{11}-c_{1} d_{21}}{d_{11} d_{22}-d_{21} d_{12}}, \\
& d_{11}=\sum_{k=1}^{n} \int_{\rho_{k}}^{\rho_{k+1}} \eta \cdot \gamma_{10}^{(k)}(\eta) d \eta \\
& d_{12}=\sum_{k=1}^{n} \int_{\rho_{k}}^{\rho_{k+1}} \eta \cdot \gamma_{20}^{(k)}(\eta) d \eta \\
& d_{21}=\sum_{k=1}^{n} \int_{\rho_{k}}^{\rho_{k+1}} \eta \cdot \gamma_{10}^{(k)}(\eta) \cdot v^{(k)}(\eta) d \eta, \\
& d_{22}=\sum_{k=1}^{n} \int_{\rho_{k}}^{\rho_{k+1}} \eta \cdot\left(\gamma_{20}^{(k)}(\eta) \cdot v^{(k)}(\eta)+E^{(k)}(\eta)\right) d \eta, \\
& c_{1}=\rho_{1}^{2} \cdot p_{1}-\rho_{n+1}^{2} \cdot p_{2}+\sum_{k=1}^{n} \int_{\rho_{k}}^{\rho_{k+1}} \eta \cdot \gamma_{00}^{(k)}(\eta) d \eta, \\
& c_{2}=\frac{p}{2 \pi}+\sum_{k=1}^{n} \int_{\rho_{k}}^{\rho_{k+1}} \eta \\
& \cdot\left(E^{(k)}(\eta) \cdot \Phi^{(k)}\left(T_{k}(\eta)\right)-\gamma_{00}^{(k)}(\eta) \cdot v^{(k)}(\eta)\right) d \eta,
\end{aligned}
$$

where: $\Phi^{i}\left(T_{i}\right)$ is the purely thermal deformation, $p_{1}, p_{2}$ are the given constant pressures (stresses) on inner $\left(\rho=\rho_{1}\right)$ and outer ( $\rho=\rho_{4}$ ) surfaces of cylinder, $p$ denotes known force loadings at the ends of the cylinder, $\delta_{i k}=\left\{\begin{array}{l}1, i=k, \\ 0, i \neq k,\end{array}\right.$ is a Kronecker symbol.

Formulas (25)-(31) to determine the stress-strain state are valid for thin layers only i.e. for which the trapezoidal formula

$\int_{\rho_{i}}^{\rho} Y(\eta) d \eta=\frac{\rho-\rho_{i}}{2}\left(Y(\rho)+Y\left(\rho_{i}\right)\right)$

is satisfied with the preset accuracy.

If the cylinder contains thin and thick layers, then each thick layer is segmented into few thinner ones made of the same material. To prove that cylinder has sufficiently thin layers the integral condition (Popovych and Kalynyak, 2014)

$\rho_{1}^{2} \cdot p_{1}-\rho_{n+1}^{2} \cdot p_{2}=\sum_{k=1}^{n} \int_{\rho_{k}}^{\rho_{k+1}} \eta \cdot \sigma^{(k)}(\eta) d \eta$

should be satisfied.

\section{PARTICULAR CASES}

Let us consider the case if the coefficients of thermal conductivity and mechanical characteristics of the non-thermosensitive cylinder (we denote them with $\mathrm{N}$ ) are equal to the basic values of corresponding components of the thermosensitive one. The temperature distribution has the form (24), where $\lambda_{t N}^{(i)}=\lambda_{t 0}^{(i)}, i=$ $\overline{1,3}$. The Poisson ratio, the coefficient of the linear thermal expansion and the elastic modulus will have the following form $v_{N}^{(i)}=$ $v_{0}^{(i)}, \alpha_{t N}^{(i)}=\alpha_{t 0}^{(i)}, E_{N}^{(i)}=E_{0}^{(i)}, i=\overline{1,3}$ and formulas for the definition of components of the stress-strain state take the form:

$$
\begin{aligned}
\sigma_{N}^{(i)}(\rho)= & \gamma_{10 N}^{(i)}(\rho) \sigma_{N}^{(1)}\left(\rho_{1}\right)+\gamma_{20 N}^{(i)}(\rho) e_{z}+\gamma_{00 N}^{(i)}(\rho), \\
\sigma_{r N}^{(i)}(\rho)= & \gamma_{1 r N}^{(i)}(\rho) \sigma_{N}^{(1)}\left(\rho_{1}\right)+\gamma_{2 r N}^{(i)}(\rho) e_{z}+\gamma_{0 r N}^{(i)}(\rho), \\
\sigma_{\varphi N}^{(i)}(\rho)= & \sigma_{N}^{(i)}(\rho)-\sigma_{r N}^{(i)}(\rho), \\
\sigma_{z N}^{(i)}(\rho)= & E_{0}^{(i)} e_{z N}+v_{0}^{(i)} \sigma_{N}^{(i)}(\rho)-E_{0}^{(i)} \Phi_{N}^{(i)}\left(T_{i}\right), \\
e_{r N}^{(i)}(\rho)= & \frac{1}{E_{0}^{(i)}}\left(\sigma_{r N}^{(i)}(\rho)-v_{0}^{(i)}\left(\sigma_{\varphi N}^{(i)}(\rho)+\sigma_{z N}^{(i)}(\rho)\right)\right. \\
& \left.+E_{0}^{(i)} \Phi_{N}^{(i)}\left(T_{i}\right)\right), \\
e_{\varphi N}^{(i)}(\rho)= & \frac{1}{E_{0}^{(i)}}\left(\sigma_{\varphi N}^{(i)}(\rho)-v_{0}^{(i)}\left(\sigma_{r N}^{(i)}(\rho)+\sigma_{z N}^{(i)}(\rho)\right)\right. \\
& \left.+E_{0}^{(i)} \Phi_{N}^{(i)}\left(T_{i}\right)\right), \\
u_{r N}^{(i)}(\rho)= & \rho \cdot e_{\varphi N}^{(i)}(\rho),
\end{aligned}
$$

$$
\begin{aligned}
& \gamma_{10 N}^{(i)}(\rho)=\frac{1}{\psi_{N}^{(i)}}\left(\frac{1-\left(v_{0}^{(1)}\right)^{2}}{E_{0}^{(1)}}+\left(1-\delta_{1 i}\right) \sum_{k=1}^{i-1} \beta_{N}^{(k)} \cdot \gamma_{1 r N}^{(k)}\left(\rho_{k+1}\right)\right), \\
& \gamma_{20 N}^{(i)}(\rho)=\frac{1}{\psi_{N}^{(i)}}\left(v_{0}^{(i)}-v_{0}^{(1)}+\left(1-\delta_{1 i}\right) \sum_{k=1}^{i-1} \beta_{N}^{(k)} \cdot \gamma_{2 r N}^{(k)}\left(\rho_{k+1}\right)\right),
\end{aligned}
$$$$
\gamma_{00 N}^{(i)}(\rho)=\frac{1}{\psi_{N}^{(i)}}\left(\left(1-\delta_{1 i}\right) \sum_{k=1}^{i-1} \beta_{N}^{(k)} \gamma_{0 r N}^{(k)}\left(\rho_{k+1}\right)-F^{(i)}(\rho)\right) \text {, }
$$$$
\gamma_{1 r N}^{(i)}(\rho)=\frac{1}{\rho^{2}}\left(\left(1-\delta_{1 i}\right) \rho_{i}^{2} \gamma_{1 r N}^{(i-1)}\left(\rho_{i}\right)+\int_{\rho_{i}}^{\rho} \eta \gamma_{10 N}^{(i)}(\eta) d \eta\right),
$$$$
\gamma_{2 r N}^{(i)}(\rho)=\frac{1}{\rho^{2}}\left(\left(1-\delta_{1 i}\right) \rho_{i}^{2} \gamma_{2 r N}^{(i-1)}\left(\rho_{i}\right)+\int_{\rho_{i}}^{\rho} \eta \gamma_{20 N}^{(i)}(\eta) d \eta\right) \text {, }
$$$$
\gamma_{0 r N}^{(i)}(\rho)=\frac{1}{\rho^{2}}\left(-\rho_{1}^{2} p_{1} \delta_{1 i}+\left(1-\delta_{1 i}\right) \rho_{i}^{2} \gamma_{0 r N}^{(i-1)}\left(\rho_{i}\right)\right.
$$$$
\left.+\int_{\rho_{i}}^{\rho} \eta \gamma_{00 N}^{(i)}(\eta) d \eta\right)
$$

$\psi_{N}^{(i)}=\frac{1-\left(v_{0}^{(i)}\right)^{2}}{E_{0}^{(i)}}, \varphi_{N}^{(i)}=\frac{1-v_{0}^{(i)}}{E^{(i)}}, \beta_{N}^{(i)}=\varphi_{N}^{(i+1)}-\varphi_{N}^{(i)}$, 


$$
\begin{aligned}
F_{N}^{(i)}(\rho)= & \left(1-v_{0}^{(i)}\right) \cdot \Phi_{N}^{(i)}\left(T_{i N}(\rho)\right) \\
& -\left(1-v_{0}^{(1)}\right) \cdot \Phi_{N}^{(1)}\left(T_{1 N}\left(\rho_{1}\right)\right),
\end{aligned}
$$

$\Phi_{N}^{(i)}\left(T_{i N}\right)=t_{0} \cdot \alpha_{t 0}^{(i)}\left(T_{i N}-T_{p}\right)$,

$\sigma_{N}^{(1)}\left(\rho_{1}\right)=\frac{c_{1 N} d_{22 N}-c_{2 N} d_{12 N}}{d_{11 N} d_{22 N}-d_{21 N} d_{12 N}}$,

$e_{z N}=\frac{c_{2 N} d_{11 N}-c_{1 N} d_{21 N}}{d_{11 N} d_{22 N}-d_{21 N} d_{12 N}}$,

$d_{11 N}=\sum_{k=1}^{n} \int_{\rho_{k}}^{\rho_{k+1}} \eta \cdot \gamma_{10 N}^{(k)}(\eta) d \eta$,

$d_{12 N}=\sum_{k=1}^{n} \int_{\rho_{k}}^{\rho_{k+1}} \eta \cdot \gamma_{20 N}^{(k)}(\eta) d \eta$,

$d_{21 N}=\sum_{k=1}^{n} \int_{\rho_{k}}^{\rho_{k+1}} \eta \cdot \gamma_{10 N}^{(k)}(\eta) \cdot v_{0}^{(k)} d \eta$,

$d_{22 N}=\sum_{k=1}^{n} \int_{\rho_{k}}^{\rho_{k+1}} \eta \cdot\left(\gamma_{20 N}^{(k)}(\eta) \cdot v_{0}^{(k)}+E_{0}^{(k)}\right) d \eta$,

$c_{1 N}=\rho_{1}^{2} \cdot p_{1}-\rho_{n+1}^{2} \cdot p_{2}+\sum_{k=1}^{n} \int_{\rho_{k}}^{\rho_{k+1}} \eta \cdot \gamma_{00 N}^{(k)}(\eta) d \eta$,

$c_{2 N}=\frac{p}{2 \pi}+\sum_{k=1}^{n} \int_{\rho_{k}}^{\rho_{k+1}} \eta$

$$
\cdot\left(E_{0}^{(k)} \cdot \Phi_{N}^{(k)}\left(T_{k N}(\eta)\right)-\gamma_{00 N}^{(k)}(\eta) \cdot v_{0}^{(k)}\right) d \eta .
$$

In case of the average integral values of coefficients of thermal conductivity:

$$
\begin{aligned}
\lambda_{t n}^{(i)}=\lambda_{t s}^{(i)} & =\frac{1}{T_{e}-T_{b}} \int_{T_{b}}^{T_{e}} \lambda_{t 0}^{(i)}\left(1+k_{i}\left(T_{i}-T_{b}\right)\right) d T_{i} \\
& =\lambda_{t 0}^{(i)}\left(1+\frac{k_{i}}{2}\left(T_{e}-T_{b}\right)\right), \\
\left\{v_{t n}^{(i)}, \alpha_{t n}^{(i)}, E_{t n}^{(i)}\right\} & =\left\{v_{t s}^{(i)}, \alpha_{t s}^{(i)}, E_{t s}^{(i)}\right\} \\
& =\frac{1}{T_{e}-T_{b}} \int_{T_{b}}^{T_{e}}\left\{v^{(i)}\left(T_{i}\right), \alpha_{t}^{(i)}\left(T_{i}\right), E^{(i)}\left(T_{i}\right)\right\} d T_{i},
\end{aligned}
$$

$i=\overline{1,3}$,

we obtain the temperature distribution (24), where

$B i_{n}=B i \frac{\lambda_{t 0}^{(3)}}{\lambda_{t s}^{(3)}}, P o_{n}=P o \frac{\lambda_{t 0}^{(2)}}{\lambda_{t s}^{(2)}}, K_{\lambda n}^{(2)}=\frac{\lambda_{t s}^{(2)}}{\lambda_{t s}^{(1)}}, K_{\lambda n}^{(3)}=\frac{\lambda_{t s}^{(3)}}{\lambda_{t s}^{(2)}}$.

The formulas for calculating the stress-strain state will have the form (34)-(40) in which the basic values of the thermomechanical characteristics should be replaced by average integral ones.

\section{NUMERICAL RESEARCH}

Let us investigate the influence of temperature dependence of the thermal and mechanical characteristics of the cylinder materials on the temperature distribution and on components of stressed-strain state at different input parameters. The material in the first and in the third layers is the ceramics $\mathrm{ZrO} 2$, and in the second one is the titanium alloy Ti-6Al-4V (Tanigava and Akai, 1996).

Experimentally specified coefficients and thermomechanical characteristics were lineary approximated (5) in the temperature range $300 \div 1100 \mathrm{~K}$ using the least squares method. The following values were established for ceramics:

$$
\begin{aligned}
\lambda_{t}^{(1,3)}=1.915 & \cdot\left(1+0.24664 \cdot\left(T_{1,3}-T_{b}\right)\right)[\mathrm{W} / \mathrm{m} \cdot \mathrm{K}], \\
v^{(1,3)}=0.333 & \\
\alpha_{t}^{(1,3)}=8.783 \cdot 10^{-6} & \cdot\left(1-1.4128 \cdot\left(T_{1,3}-T_{b}\right)+1.7496\right. \\
& \left.\cdot\left(T_{1,3}-T_{b}\right)^{2}\right)[1 / \mathrm{K}], \\
E^{(1,3)}=116.381 & \cdot\left(1-0.521357 \cdot\left(T_{1,3}-T_{b}\right)-0.084215\right. \\
& \left.\cdot\left(T_{1,3}-T_{b}\right)^{2}\right)[\mathrm{GPa}],
\end{aligned}
$$

and for titanium alloy

$$
\begin{aligned}
\lambda_{t}^{(2)}= & 6.2 \cdot\left(1+3.016 \cdot\left(T_{2}-T_{b}\right)\right)[\mathrm{W} / \mathrm{m} \cdot \mathrm{K}], \\
v^{(2)}= & 0.2984 \cdot\left(1+0.118 \cdot\left(T_{2}-T_{b}\right)\right), \\
\alpha_{t}^{(2)}= & 8.8559 \cdot 10^{-6} \\
& \cdot\left(1+0.49014 \cdot\left(T_{1,3}-T_{b}\right)-0.36754\right. \\
& \left.\cdot\left(T_{1,3}-T_{b}\right)^{2}\right)[1 / \mathrm{K}] \\
E^{(2)}= & 105.05 \cdot\left(1-0.5916 \cdot\left(T_{1,3}-T_{b}\right)\right)[\mathrm{GPa}] .
\end{aligned}
$$

The coordinates of layer boundaries in the cylinder are equal $\rho_{1}=0.6, \rho_{2}=0.75, \rho_{3}=0.95, \rho_{4}=1$ with characteristic length $l_{0}=r_{4}$. The dimensionless values of temperature are $T_{b}=3 / 11, T_{e}=1, T_{\text {in }}=1 / 3, T_{\text {out }}=1 / 2$, Pomerantsev and Biot numbers are $\mathrm{Po}=-7, \mathrm{Bi}=2$. The constant pressures on inner and outer surfaces of cylinder and axial loadings at the ends are $p_{1}=p_{2}=p=0$.

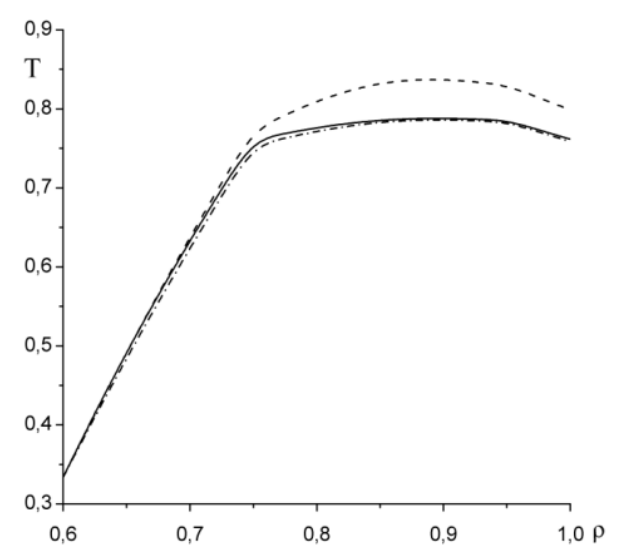

Fig. 1. The temperature distribution

The temperature distribution of the considered cylinder is presented in Fig. 1. Here and below the solid line corresponds to the thermosensitive cylinder, dashed line corresponds to the nonthermosensitive cylinder with basic values, dash-dotted line corresponds to the non-thermosensitive cylinder with the average integral values of the coefficient of thermal conductivity.

The maximal difference between temperatures in thermosen- 
sitive and non-thermosensitive (with basic and average integral values of the coefficient of thermal conductivity) cylinders do not exceed $6 \%$.

The algorithm for determining the components of stress-strain state because the formulas (25)-(31) are valid for thin layers only has following steps:

1. Calculating formulas (25)-(31) without segmentation of each layer in the cylinder into thin pieces.

2. Checking the satisfying of the integral condition (33).

3. If the condition (33) is not satisfied then the number of segments will be increased. Then we return to step 2. Otherwise the calculation is over.

Tab. 1 presents data obtained in finding sufficient segmentation of layers into components to achieve the accuracy $\varepsilon=10^{-4}$.

Tab. 1. The result of segmentation of cylinder layers into thin components

\begin{tabular}{|c|c|c|c|c|}
\hline \multirow{2}{*}{$\begin{array}{c}\text { The total } \\
\text { number } \\
\text { of layers }\end{array}$} & \multicolumn{3}{|c|}{$\begin{array}{c}\text { The number of segmentation } \\
\text { in each layer }\end{array}$} & $\begin{array}{c}\text { The satisfying of } \\
\text { integral conditions }\end{array}$ \\
\cline { 2 - 4 } & I layer & II layer & III layer & \\
\hline 3 & 1 & 1 & 1 & -0.004015 \\
\hline 4 & 1 & 2 & 1 & -0.002988 \\
\hline 5 & 2 & 2 & 1 & -0.000994 \\
\hline 6 & 2 & 3 & 1 & -0.000832 \\
\hline
\end{tabular}

As we can see the representation of the considered cylinder as the 6 components body is enough to achieve the preset accuracy. Fig. 2-7 show distributions of stresses, deformations and displacements. The constant axes deformation is $\mathrm{e}_{\mathrm{z}}=0.003907$.

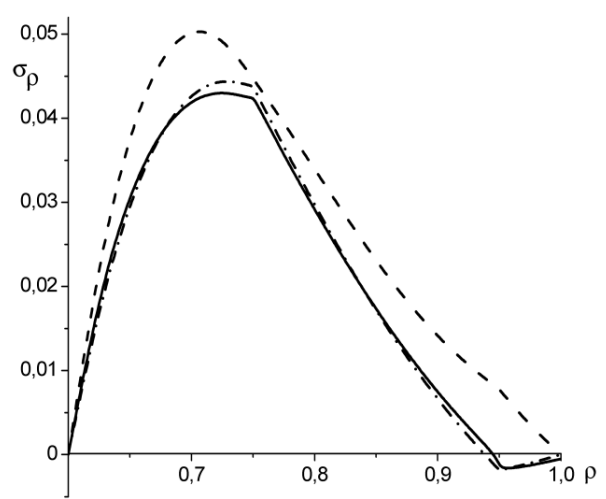

Fig. 2. The radial stresses

From Fig. 2, 3, 5-7 we can note that the stress-strain components distribution in the nonthermosensitive cylinder with average integral values of mechanical characteristics gives a better approximation to the same distribution in thermosensitive hollow cylinder in comparison to the stress-strain components distribution in the nonthermosensitive cylinder with basic values. In particular, the maximum differences of total stresses are $18 \%$, of radial stresses are $8 \%$, of circumferential stresses are $15 \%$, of radial deformation are $10 \%$, of radial displacements are $10 \%$.

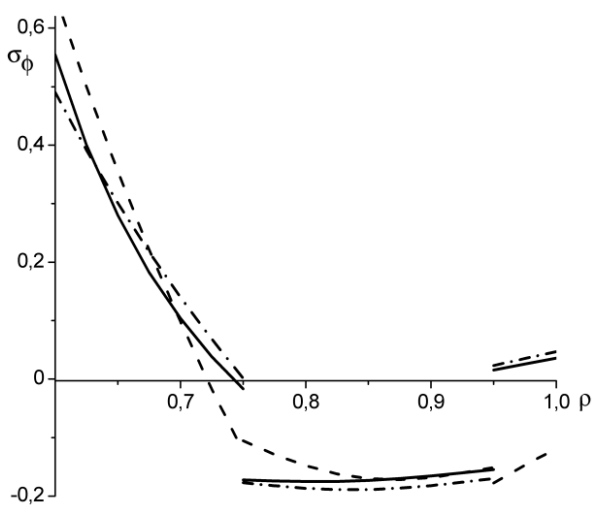

Fig. 3. The circumferential stresses

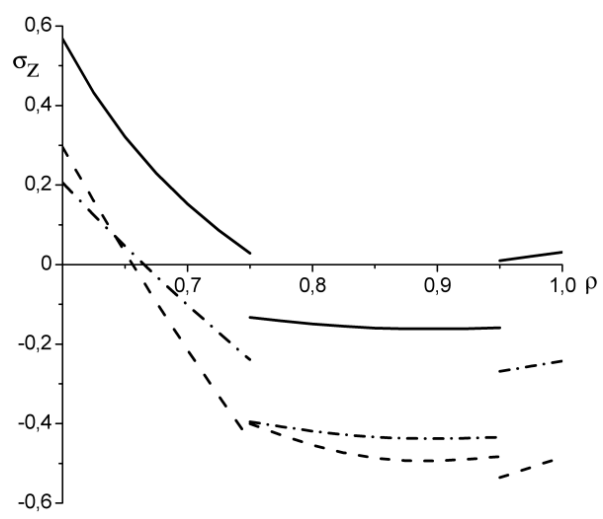

Fig. 4. The axial stresses

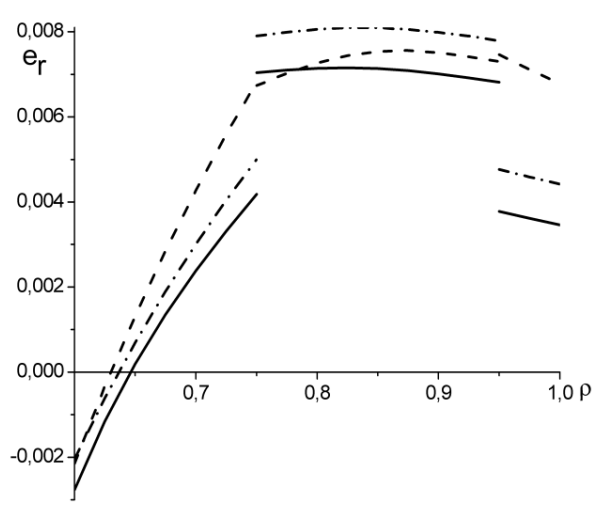

Fig. 5. The radial deformation

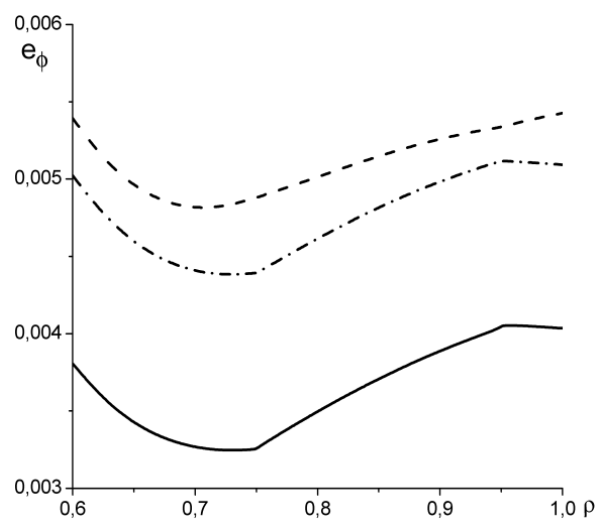

Fig. 6. The circumferential deformation 


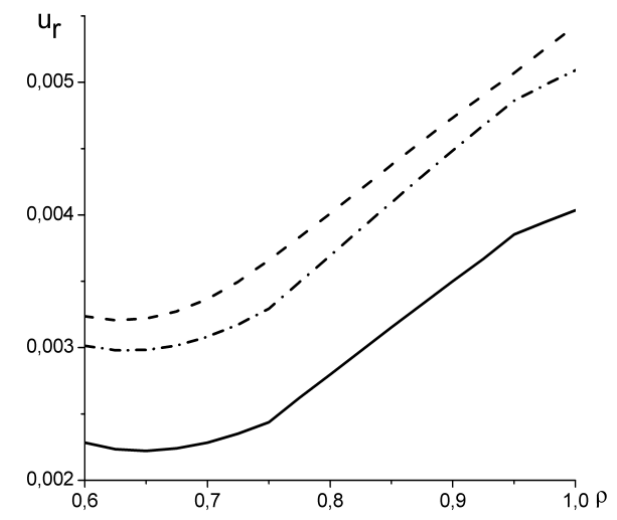

Fig. 7. The radial displacements

Use of model of nonthermosensitive body with basic values of its thermomechanical characteristics gives no correct approximation for such distribution of appropriate stress-strain components of thermosensitive cylinder but also leads to different quality results in some cases. Thus, in Fig. 2-4 we can see that stresses in the third layer of nonthermosensitive cylinder (with basic thermomechanical characteristics) are changed not only quantitatively, but also inherently (the compression in thermosensitive one and stretching in nonthermosensitive or conversely).

Let us note that for determining axial stresses it is necessary to take into account the thermosensitivity of material components because the use of basic and average integral values of thermomechanical characteristics will not give the correct distribution of $\sigma_{\mathrm{z}}$.

\section{SUMMARY}

The nonlinear mathematical model of temperature distribution in a infinite three-layer hollow thermosensitive cylinder with heat sources distributed by parabolic law in the second layer, constant temperature and convective heat exchange on the boundary surfaces has been created. The components of the stress-strain state have been determined.

If to neglect the depemdence of thermomechanical characteristics of material components (the replacement for basic or average integral ones), then the large differences between distributions of components of stress-strain state of thermosensitive and nonthermosensitive cylinders may appear and lead to qualitatively different distributions. For the selected layer materials was shown that the distribution of the stress-strain state in nonthermosensitive cylinder with average integral values of mechanical characteristics gives a better approximation to such distribution in thermosensitive hollow cylinder. This can be useful for its rapid estimation.

\section{REFERENCES}

1. Argeso H., Eraslan A. N. (2008), On use of temperature-dependent physical properties in thermomechanical calculations for solid and hollow cylinders, International journal of thermal sciences, 47(2), 136-146.

2. Aziz A., Torabi M. (2013), Thermal stresses in a hollow cylinder with convective boundary conditions on the inside and outside surfaces, Journal of thermal stresses, 36(10), 1096-1111.
3. Bazarenko N. (2008), The contact problem for hollow and solid cylinders with stress-free faces, Journal of applied mathematics and mechanics, 72(2), 214-225.

4. Carslaw H. S., Jaeger J. C. (1959), Conduction of heat in solids, Oxford: Clarendon Press.

5. Fazeli H., Abdous A., Karabi H., Moallemi N., Esmaeili M. (2013), Analysis of transient heat conduction in a hollow cylinder using Duhamel theorem, International journal of thermophysics, 34(2), 350-365.

6. Goto T., Suzuki M. (1996), A boundary integral equation method for nonlinear heat conduction problems with temperature dependent material properties, Internationla journal of heat and mass transfer, 39(4), 823-830.

7. Grzes P. (2010) Influence of thermosensitivity of materials on the temperature of a PAD/DISC systems, Acta Mechanica et Automatica, 5(4), 46-53.

8. Kushnir R. M., Popovych V. S. (2009), Thermoelasticity of thermosensitive solids, Spolom, Lviv (in Ukrainian).

9. Kushnir R. M., Popovych V. S. (2011), Heat Conduction Problems of Thermosensitive Solids under Complex Heat Exchange, In Tech.

10. Kushnir R. M., Protsiuk Yu. B. (2010), Thermoelastic State of Layered Thermosensitive Bodies of Revolution for the Quadratic Dependence of the Heat-Conduction Coefficients, Materials Science, 46(1), 1-15.

11. Lee H.-J. (1998), The effect of temperature dependent material properties on the response of piezoelectric composite materials, Journal of Intelligent Material Systems and Structures, 9(7), 503-508.

12. Noda N. (1991), Thermal Stresses in Materials with TemperatureDependent Properties, Applied Mechanics Reviews, 44(9), 383-397.

13. Och E. (2014), Frictional heating of sliding semi-spaces with simple thermal nonlinearities, Acta Mechanica et Automatica, 7(4), 236-240.

14. Och E. (2015), Frictional heating during sliding of two semi-spaces with arbitrary thermal nonlinearity, Acta Mechanica et Automatica, 8(4), 204-208.

15. Popovych V. S., Kalynyak B. M. (2014), Mathematical modeling and method of determining the static thermoelastic state of multilayered thermosensitive cylinders, Math. Methods and Phys.Mech. Fields, 57(2), 169-186 (in Ukrainian).

16. Shariyat M. (2007), Thermal buckling analysis of rectangular composite plates with temperature dependent properties based on a layerwise theory, Thin-Walled Structures, 45(4), 439-452.

17. Shen H.-S. (2001), Thermal postbuckling behavior of imperfect shear deformable laminated plates with temperature-dependent properties, Computer Methods in Applied Mechanics and Engineering, 190(4041), 5377-5390.

18. Shi Zh., Zhang T., Xiang H. (2007), Exact solutions of heterogeneous elastic hollow cylinders, Composite structures, 79(1), 140-147.

19. Tanigawa Y., Akai T. (1996) Transient heat conduction and thermal stress problems of a nonhomogeneous plate with temperaturedependent material properties, Journal of Thermal Stresses, 19(1), 77-102.

20. Vigak V. (1999), Solutions of one-dimensional problems of elasticity and thermoelasticity for cylindrical piecewise homogeneous bodies, Journal of Mathematical Science, 96(2), 3056-3064.

21. Yevtuchenko A. A., Kuciej M., Och E. (2014), Influence of thermal sensitivity of the pad and disk materials on the temperature during braking, International Communications in Heat and Mass Transfer, 55, 84-92.

22. Zhu X. K., Chao Y. U (2002), Effect of temperature-dependent material properties on welding simulation, Computers \& Structures, 80(11), 967-976.

The work has been accomplished and partly financed under the project No. 0114 U005081 of National Academy of Sciences of Ukraine. 